\title{
State of Freshwater Eels in the South Pacific
}

\author{
Anushka Hewavitharane* \\ Kyushu University, Japan
}

*Corresponding author: Anushka Hewavitharane, Kyushu University, Japan.

\section{Editorial}

Freshwater eels of the genus Anguilla consists of 16 species and 3 subspecies that are distributed in the Indo-Pacific and North Atlantic Ocean. They are consumed as delicacies throughout their dispersal range especially in Japan, Europe and the Americas. With growing demand for eels on the international market the three major species currently cultured in the aquaculture industry, Japanese eels $A$. japonica, European eel $A$. anguilla and American eel $A$. rostrata have all been severely overfished with populations on the brink of extinction.

This has resulted in an increased interest from Southeast Asian eel aquaculturists in procuring anguillid glass eels and elvers from the South Pacific which is the last frontier for eel stocks. But with little or no knowledge of inshore migration mechanisms, seasonality or abundance of glass eels in the South Pacific region there is a grave risk of overexploitation.

Although there are approximately thirteen of the total nineteen species occurring in the tropical Pacific Oceans with 6 species distributed within the islands and landmasses of the South Pacific there is very little knowledge on the exact population density of all six species. This may be due to the fact that most Island nations in the South Pacific have vast numbers of Islands scattered within each Island territory but most importantly there was never a need to undertake stock assessments of freshwater eels as they did not have a price tag attached to them until now. The tropical anguillid eel population in the South Pacific seems to be high in diversity but low in abundance but again with very scarce information it is extremely difficult to quantify population statistics. Therefore there is an urgency for scientists and biologists in the aquatic sciences to undertake research and broaden the knowledge on freshwater eels in the South Pacific.

Furthermore it is unfair to label a highly valuable income generating enterprise such as the eel aquaculture industry as a scourge in the Pacific especially as it has the potential of improving the livelihorod of poor Pacific communities. But first there needs more knowledge on freshwater eels needs to be garnered and with this information promulgation of regulations and laws governing the effective utilization of eel stocks be put in place to prevent the negative effects as seen with the Japanese, European and American eels. 